\title{
Integrated Technology Platform Protein Kinases for Drug Development in Oncology
}

\author{
Christoph Sachsenmaier and Christoph Schäechtele \\ ProQinase GmbH, Freiburg, Germany
}

BioTechniques 33:S101-S106 (October 2002)

\section{ABSTRACT}

Protein kinases are among the most promising targets for drug discovery and development, mostly in oncology but also in other fields such as inflammation, Alzheimer's, and infectious diseases. The Integrated Technology Platform Protein Kinases was designed as a comprehensive tool for drug discovery in the field of oncology. It combines modules for the identification and validation of novel target protein kinases, a unique panel of active recombinant protein kinases, high-throughput screening, selectivity profiling, cellular testing, and in vivo tumor models. Here we give an overview of the Integrated Technology Platform Protein Kinases as well as data that validate each module.

\section{HISTORY AND BACKGROUND}

Cellular protein kinases are important constituents of signal transduction pathways regulating all fundamental biological processes, including proliferation, growth, migration, metabolism, and death. Protein kinases are classified as serine/threonine- or tyrosine-specific based on their substrate specificity. Early studies of protein kinase function led to the search for specific protein kinase inhibitor molecules. One of the first protein kinase inhibitors, staurosporine (an alkaloid from Streptomyces staurosporeus), and its derivatives are widely used to study the role and functions of phospholipid- and calcium-dependent protein kinases.

Not long after the identification that viral oncogenes in animals are protein kinases (e.g., v-src and v-abl), it became apparent that some human diseases may also have their origin in altered cellular protein kinase activity. This made searching for specific protein kinase inhibitor molecules a prime goal of drug discovery, especially in oncology. However, many doubts existed (and still do). Is it possible to identify specific protein kinase inhibitors, as most inhibitor molecules bind to the very conserved ATP-binding site in protein kinases? Would there be unbearable side effects of nonspecific protein kinase inhibitor drugs, given the normal role that the targeted protein kinases play in the human body?

Despite these legitimate concerns, the search for protein kinase inhibitors as potential drugs is one of the most ambitious drug development projects in pharmaceutical and biotech com- panies worldwide. Antibodies or other highly target-specific molecules (such as antisense RNA) are used to circumvent the specificity issue. Other more or less specific chemical inhibitor molecules have also been developed that, although targeting the ATP binding site, display remarkable kinase specificity. However, selectivity profiling of compounds against a broad range of other kinases has not been thoroughly pursued in the past, mostly because of the lack of available active recombinant protein kinases and suitable assay technologies.

Two decades of effort have culminated in one protein kinase inhibitor drug for clinical use in oncology (Glivec $^{\mathrm{TM}}$, Gleevec ${ }^{\mathrm{TM}}$; Novartis Pharma, Basel, Switzerland). This first-generation drug shows promise but also demands greater effort in generating new and improved protein kinase inhibitors (mainly in terms of efficacy and in overcoming the development of resistance). Successful drug discovery and development targeting protein kinases has two major prerequisites: (i) a set of well-characterized and therapeutically validated protein kinase targets and ( $i i)$ a broad as possible portfolio of protein kinases at hand to determine the specificity of potential protein kinase inhibitors. The draft sequence of the human genome puts the number of potential protein kinase sequences between 600 and 800 to date. Here we describe our efforts toward meeting these two basic prerequisites and the additional features that make up the Integrated Technology Platform Protein Kinases.

\section{IDENTIFICATION OF NOVEL PROTEIN KINASE TARGETS IN ONCOLOGY}

Figure 1 outlines our strategy for the identification and validation of novel target protein kinases. Three different experimental approaches are followed to identify novel target protein kinases relevant to human tumors. Differential proteomics approaches demand the availability of sufficient biological material. Human biopsies usually do not contain enough protein to successfully detect proteins that are expressed at low levels (like protein kinases). Therefore, methods to separate and enrich the kinase-subproteome are very important. We have developed a PanKinase affinity matrix based on a kinase inhibitor molecule that in our hands inhibits the activity of all protein kinases test- 
Table 1. List of Established Recombinant Protein Kinases

\begin{tabular}{|c|c|c|c|c|}
\hline \multicolumn{2}{|c|}{ Receptor Tyrosine Kinases } & \multicolumn{2}{|c|}{ Serine/Threonine Kinases } & \multirow{2}{*}{$\frac{\text { Soluble Tyrosine Kinases }}{A B L 1(h)}$} \\
\hline EGF-R (h) & MET (h) & AKT1 (h) & PKC-alpha (m) & \\
\hline ERBB2 (h) & & AKT3 (h) & PKC-beta1 (h) & \\
\hline \multirow[t]{2}{*}{ ERBB4 (h) } & NSK2 (m) & Aurora-A (h) & PKC-beta2 (h) & BRK (h) \\
\hline & & Aurora-B (h) & PKC-delta (r) & \\
\hline \multirow[t]{2}{*}{ EPHB4 (h) } & PDGFR-alpha (h) & & PKC-epsilon (m) & FAK (h) \\
\hline & PDGFR-beta (h) & CDK1/CycB (h) & PKC-eta $(\mathrm{m})$ & \\
\hline FGF-R1 (h) & & CDK2/CycA (h) & PKC-gamma (r) & JAK2 (m) \\
\hline FGF-R3 (h) & TIE2 (h) & CDK2/CycE (h) & PKC-iota (h) & \\
\hline \multirow[t]{2}{*}{ FGF-R4 (h) } & & CDK4/CycD1 (h) & PKC-mu (h) & WEE1 (h) \\
\hline & VEGF-R1 (h) & CDK6/CycD1 (h) & PKC-theta (h) & \\
\hline IGF1-R (h) & VEGF-R2 (h) & & PKC-zeta (h) & \\
\hline \multirow[t]{2}{*}{ INS-R (h) } & VEGF-R3 (h) & CK2 (h) & & \\
\hline & & & RAF1 (h) & \\
\hline \multirow[t]{5}{*}{ KIT (h) } & & $\mathrm{COT}(\mathrm{h})$ & & \\
\hline & & & S6K (h) & \\
\hline & & PCTAIRE1 (h) & & \\
\hline & & & TSF1 (h) & \\
\hline & & PLK1 (h) & & \\
\hline
\end{tabular}

ed so far. Preliminary data using recombinant serine/threonine as well as tyrosine kinases mixed into cellular lysates, as well as attempts to purify endogenous protein kinases, have yielded promising results. Currently, the efficacy of the affinity matrix

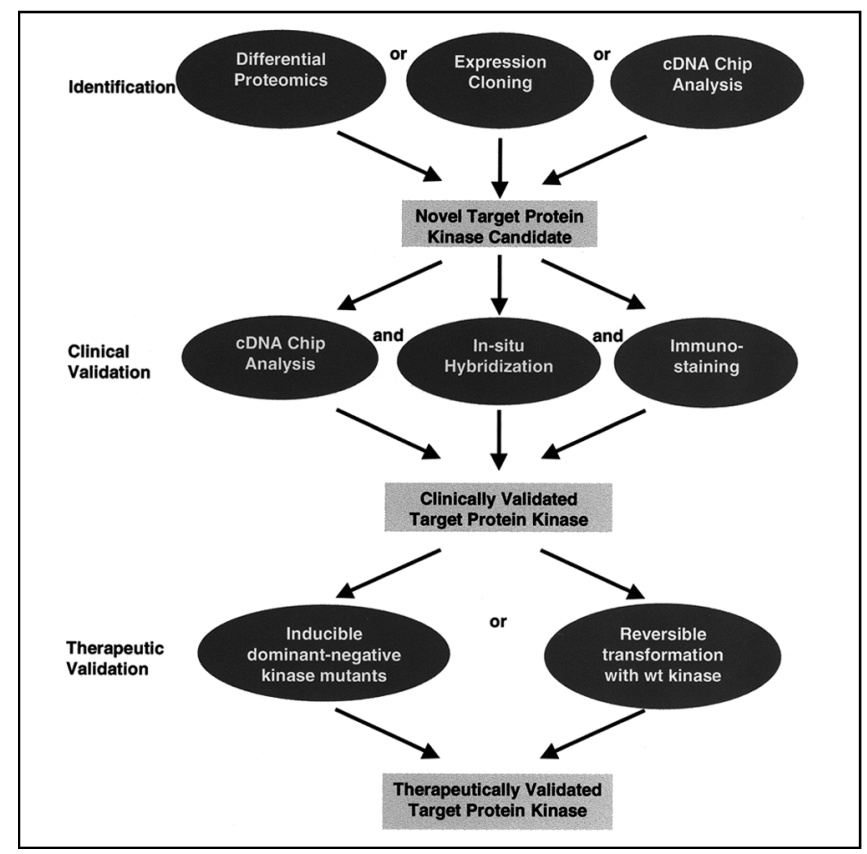

Figure 1. Schematic overview of target identification and validation strategies. toward a variety of endogenous protein kinases is being tested. Additional protein kinase inhibitors are being evaluated as ligands for affinity matrices to cover potential non-binders to the PanKinase affinity matrix.

Our expression cloning strategy aims at cloning kinase sequences that are important for the survival of cells. Cell lines that inducibly express an apoptosis-inducing factor are co-trans-

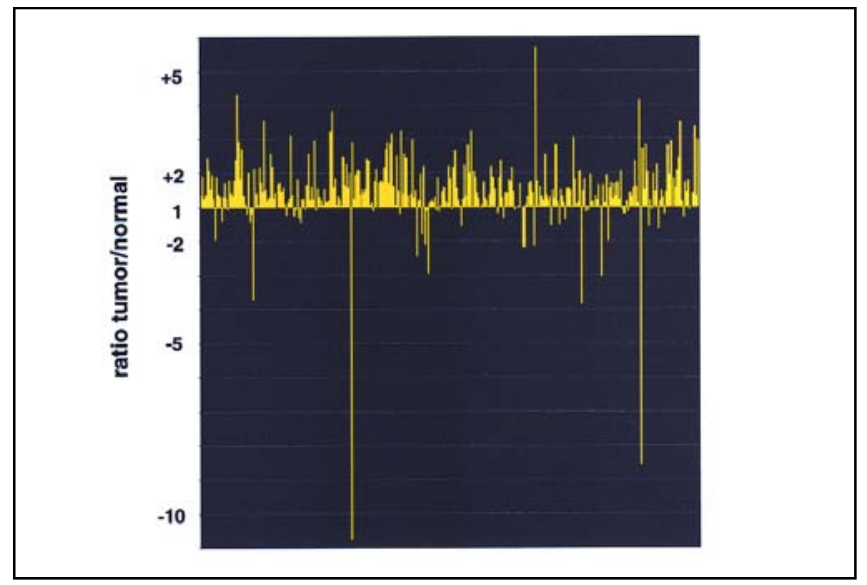

Figure 2. Expression pattern of 317 protein kinases in a matched human colon cancer sample. Matched tissue samples (tumor, healthy) from one human patient were prepared for cDNA chip analysis according to the company's instructions (Mergen Ltd., San Leandro, CA, USA). The expression levels of 317 protein ki nase sequences are depicted as ratios of expression in tumor/expression in normal tissue. Positive values indicate overexpression of a protein kinase in the tumor. 
fected with cDNA libraries from tumor cell lines, and survivor clones are analyzed for kinase-like sequences. This approach should yield kinase sequences relevant for increased survival of cells and hence potential targets for tumor therapy.

Crucial to the cDNA chip approach is access to human clinical tumor samples through a close collaboration with the University Hospital, Freiburg (Department of General Surgery). Matched pairs of samples from various human tumors and corresponding healthy tissue are being classified and processed for cDNA chip analysis (currently colon, kidney, prostate, and breast). Figure 2 shows the expression pattern of 317 protein kinases in one matched human colon sample. The graph depicts the ratio of expression between tumor/normal (positive bars meaning overexpression of a kinase in the tumor). This type of analysis allows for the rapid detection of over- and underexpressed protein kinases in tumors versus normal tissue.

\section{VALIDATION OF CANDIDATE TARGET PROTEIN KINASES}

The target validation process at ProQinase is separated into two consecutive steps, clinical and therapeutic validation (Figure 1).

For the clinical validation, the availability of larger numbers of matched human samples is necessary. To obtain statistically significant results, more than 10 samples from one tumor type should be analyzed. So far, 15 classified matched colon carcinoma samples are available to ProQinase. These are being analyzed using three different detection methods, cDNA chips, RNA in situ hybridization, and immunostaining. Antibodies are used in the immunostaining that detect specific phosphorylated isoforms of the kinase that give hints as to the activation status of a given protein kinase.

It is our conviction that it is necessary to test the therapeutic significance of a given candidate protein kinase target in an established tumor. In our opinion, this step is not often taken in most drug development programs to date. Two alternative strategies are being followed: ( $i$ ) the inducible expression of dominantnegative kinase mutants in human tumor xenografts in nude mice and (ii) the reversible transformation of mouse embryo fibroblasts by wild-type protein kinase sequences followed by transplantation into nude mice. The first approach demands the availability of a validated dominant-negative mutant of the protein kinase to be tested and has the advantage of evaluating the therapeutic significance of the protein kinase in a human tumor tissue. The second approach does not require an established kinase mutant, but rather yields the additional information that a given kinase is oncogenic (in mice) in the first place. Both strategies have been established at ProQinase, and together they yield information about whether the inhibition of a given kinase makes therapeutic sense after the tumor has been established to a visible size in mice. Currently, seven target protein kinases are under therapeutic validation using these methods.

\section{RECOMBINANT PROTEIN KINASES}

Over the past several years, a broad portfolio of recombinant protein kinases has been built up and is continuously being expanded. Protein kinases are primarily selected from the field of oncology. Table 1 shows a list of established protein kinases (currently 50). The expansion of this portfolio is an ongoing process, and approximately 120 more protein kinase sequences

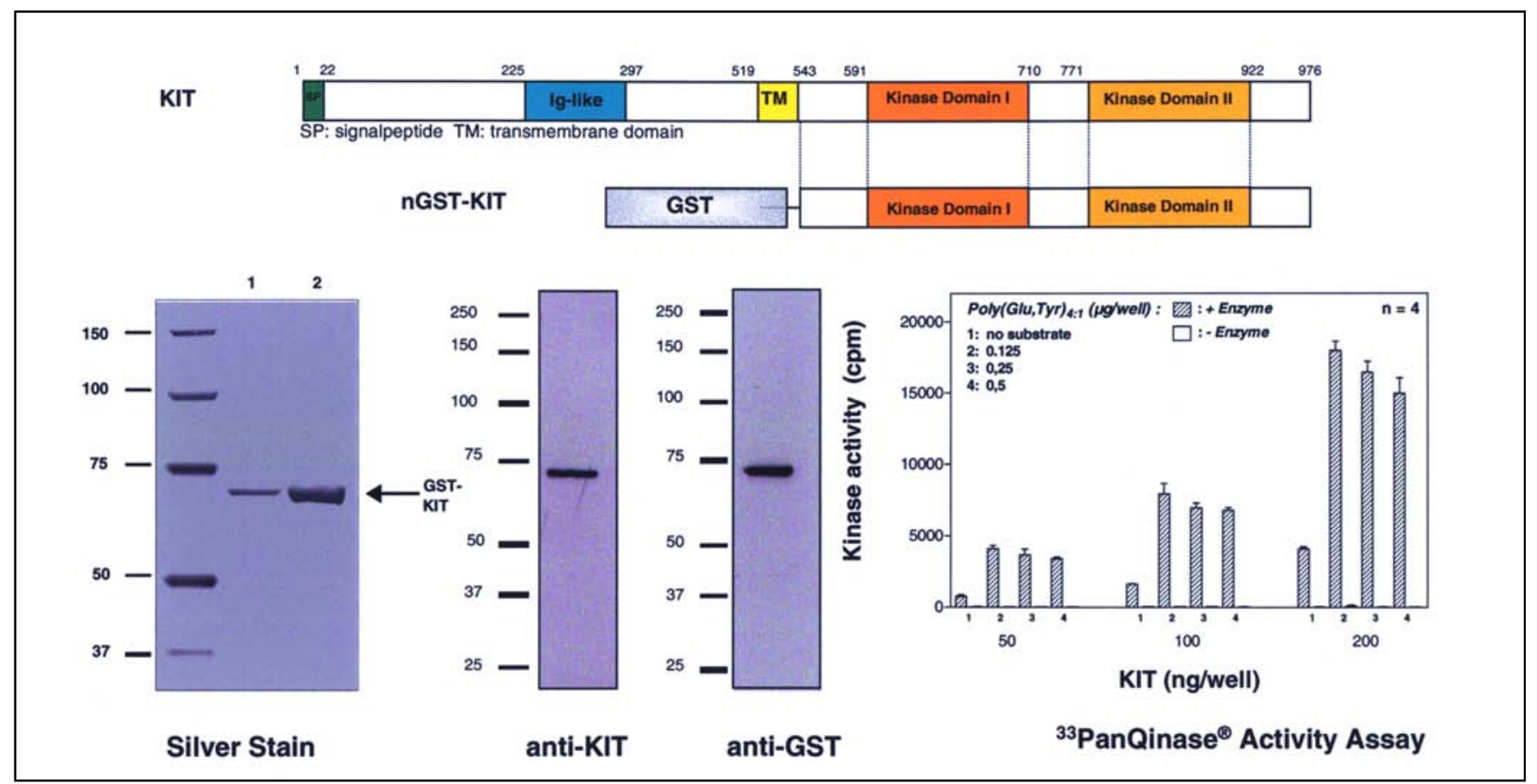

Figure 3. Expression and analysis of the KIT receptor tyrosine kinase. Schematic representation of a fusion protein containing GST and the cytoplasmic kinase domain of the KIT receptor tyrosine kinase. Affinity-purified material was analyzed by SDS-PAGE/silver stain, Western blot using a KIT- and GST-specific antibody, and by the 33 PanQinase Activity Assay. 


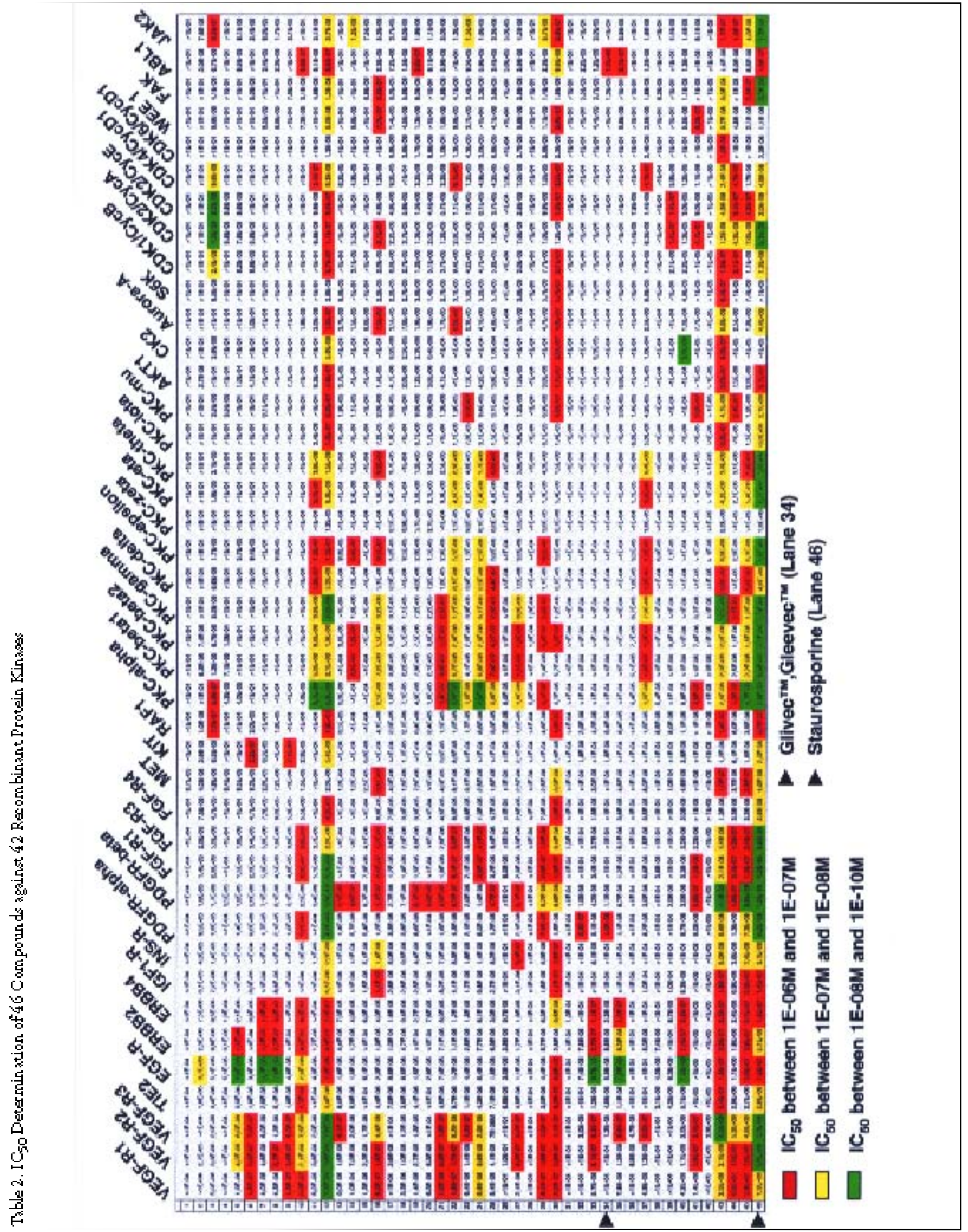


Table 3. List of Cell Lines Used for Cellular Testing of Protein Kinase Inhibitors

\begin{tabular}{|ccc|}
\hline $\begin{array}{c}\text { Receptor Tyrosine } \\
\text { Kinase }\end{array}$ & Cell Line & Transfected? \\
\hline PDGF-R & NIH-3T3 & no \\
VEGF-R2 & CHO & yes \\
IGF1-R & MCF7 & no \\
& MEF/TetOFF-IGF1R & yes \\
EGF-R & A431 & no \\
TIE2 & CHO & yes \\
\hline
\end{tabular}

Table 4. List of Established In Vivo Tumor Models

\begin{tabular}{|lcc|}
\hline \multicolumn{3}{|c|}{ Nude Mouse Xenograft (s.c.) } \\
HT-29 & Tumor & Species \\
RKO & colon & human \\
Caco-2 & colon & human \\
DU-145 & colon & human \\
A549 & prostate & human \\
N417 & lung & human \\
MCF-7 & lung & human \\
MDA-MB-231 & breast & human \\
A375 & breast & human \\
SK-OV 3 & melanoma & human \\
C6 & ovarian & human \\
MEFs & glioblastoma & rat \\
\multicolumn{4}{c|}{ transformed with target } & mouse \\
& protein kinase & \\
Name & Orthotopic-Metastasizing (Balb/c) \\
\hline Renca & Tumor & Species \\
\multicolumn{2}{|c|}{ Orthotopic-Metastasizing (Nude mouse) } \\
Name & kidney & Species \\
\hline Alexander & liver & human \\
\hline \multicolumn{4}{c}{} & & mouse \\
\hline
\end{tabular}

are currently in various stages of completion. All protein kinases are cloned from human cDNA libraries (if possible) and expressed as fusion proteins in insect cells. Affinity-purified material is checked by SDS-PAGE/silver stain, Western blot, and the proprietary 33 PanQinase ${ }^{\circledR}$ Activity Assay (Figure 3).

\section{PANQINASE ACTIVITY ASSAY}

The ${ }^{33}$ PanQinase Activity Assay is a proprietary method that allows for the determination of kinase activity of both serine/threonine and tyrosine protein kinases. It is based on the incorporation of ${ }^{33} \mathrm{P}$ into substrates, followed by the immobilization of the substrate, washing, and subsequent measurement of incorporated radioactivity. All steps are performed in 96- or 


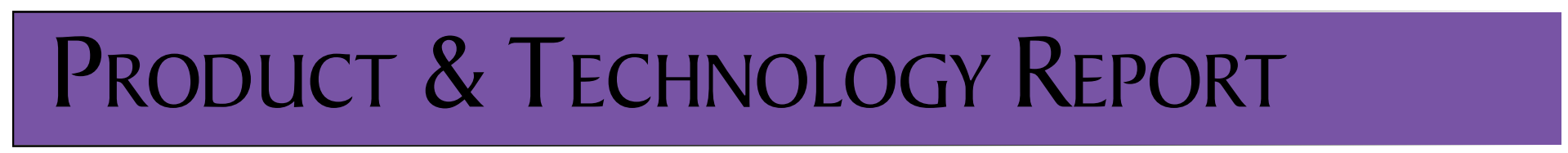

A

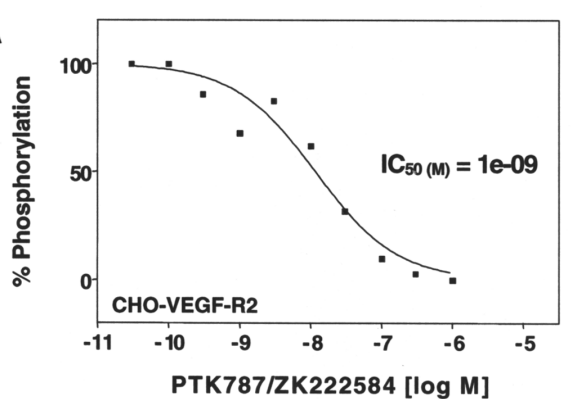

B

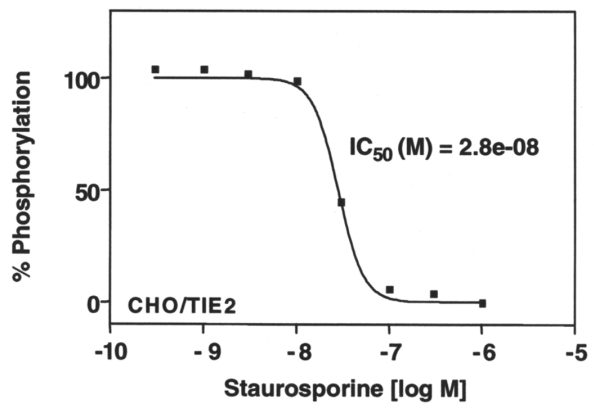

Figure 4. Cellular $\mathrm{IC}_{50}$ determination of protein kinase inhibitors. $\mathrm{CHO}$ cells overexpressing the receptor tyrosine kinases VEGF-R2 (A) and TIE2 (B) were treated with the indicated protein kinase inhibitors at 10 concentrations for 90 $\mathrm{min}$, followed by stimulation with their respective ligand for $3 \mathrm{~min}$ (A) or a nonphysiologic agent for $7 \mathrm{~min}$ (B). Cell lysates were prepared, and receptor autophosphorylation was determined using a sandwich ELISA method using a receptor-specific capture antibody followed by incubation with a phosphotyrosine-specific antibody. Percent inhibition of receptor autophosphorylation was calculated relative to untreated control cells.

384-well Flashplates ${ }^{\circledR}$ (Perkin Elmer Life Sciences, Boston, MA, USA) and are amenable to automation [we use a Beckman Coulter Sagian ${ }^{\mathrm{TM}}$ robot system (Fullerton, CA, USA)].

\section{HIGH-THROUGHPUT SCREENING AND SELECTIVITY PROFILING}

The 33PanQinase Activity Assay allows for the rapid and efficient profiling of chemical substances against the entire range of 50 active recombinant protein kinases. Table 2 shows a typical result of a profiling project where 46 compounds were tested against 42 recombinant protein kinases. Complete $\mathrm{IC}_{50}$ determination was performed for each compound using each kinase (one $\mathrm{IC}_{50}$ curve covering 10 concentrations of test compound). Several controls were included, including staurosporine (lane 46) and Glivec/Gleevec (lane 34). As can be seen for Glivec/Gleevec, the expected kinase inhibition profile was reproduced with the 33PanQinase Activity Assay. IC 50 values for the ABL, KIT, and PDGF receptors were between 1.0 and $1.8 \times 10^{-6} \mathrm{M}$, whereas the other 39 protein kinases showed at least 10 -fold higher $\mathrm{IC}_{50}$ values. The in vitro $\mathrm{IC}_{50}$ values for the three target kinases using the 33PanQinase Activity Assay were roughly 10-fold higher than those obtained in cellular assays (1). Given the different assay methods and environments (substrate phosphorylation in vitro vs. autophosphorylation in cells), this difference seems acceptable. Also, several other published $\mathrm{IC}_{50}$ values were reproduced using the 33PanQinase Activity Assay that were previously obtained using other assays and other enzyme sources (unpublished results).

\section{CELLULAR TEST SYSTEMS}

Both cellular and in vivo test systems for the evaluation of hits from the in vitro screening and profiling steps were established. Activation of cellular receptor tyrosine kinases is determined using ELISA methods. Five cellular receptor tyrosine kinase test systems have been established. The cells that are being used either "naturally" express the kinase at high levels (MCF7, NIH3T3) or are designed to overexpress the kinase of interest (Table 3). Figure 4 shows a typical result of a cellular $\mathrm{IC}_{50}$ determination using VEGF-R2 and TIE2 overexpressing cell lines.

\section{IN VIVO TUMOR MODELS}

Several mouse tumor models have been established to test candidate protein kinase inhibitors in vivo. These include human tumor cell line xenografts as well as two orthotopic tumor models (Table 4). In addition, mouse embryo fibroblasts overexpressing candidate novel protein kinase targets that have been established through our target validation efforts are being used as test models for potential kinase inhibitors directed against the given protein kinase.

The Integrated Technology Platform Protein Kinases combines all the steps of early drug discovery and development for targeting protein kinases in oncology. We believe that the sound therapeutic validation of novel protein kinase targets together with one of the world's broadest portfolio of recombinant protein kinases will allow ProQinase and its partners to identify novel nextgeneration protein kinase inhibitor drugs rapidly and efficiently.

\section{REFERENCE}

1.Capdeville, R., E. Buchdunger, J. Zimmermann, and A. Matter. 2002. Glivec (STI571, Imatinib), a rationally developed, targeted anticancer drug. Nat. Rev. Drug Disc. 1:493-502.

\section{Address correspondence to:}

Dr. Christoph Sachsenmaier

ProQinase GmbH

Breisacher Strasse 117

D-79106 Freiburg, Germany

e-mail: c.sachsenmaier@proqinase.com 